\title{
ATLAS Higgs Physics Prospects at the High Luminosity LHC
}

Thomas Koffas ${ }^{* \dagger}$

Carleton University

E-mail: Thomas.Koffasecern.ch

The High-Luminosity Large Hadron Collider will provide an unprecedented opportunity to study the properties of the Higgs boson and eventually probe for new physics beyond the Standard Model. The large anticipated data sample will allow for more precise investigations of topics already studied with earlier data samples, as well as for studies of processes that are accessible only with the much larger statistics. Rates and signal strengths will be measured for a variety of Higgs boson production and decay modes, allowing extraction of the Higgs boson couplings. Particular final states will allow differential cross-sections to be measured for all production modes, and for studies of the Higgs boson width and CP properties, as well as the tensor structure of its coupling to vector bosons. An important part of the High-Luminosity LHC experimental program will be investigations of the Higgs boson self-coupling, which is accessible via studies of di-Higgs production. In this note the projections of the ATLAS physics reach in the Higgs sector at the High-Luminosity LHC are discussed and some selected analyses are presented.

38th International Conference on High Energy Physics 3-10 August 2016

Chicago, USA

* Speaker.

${ }^{\dagger}$ On behalf of the ATLAS Collaboration. 


\section{Introduction}

The ATLAS and CMS collaborations at the Large Hadron Collider (LHC) announced the discovery of a Higgs-like boson in the summer of 2012[1],[2]. Since then, both collaborations have measured the mass of the particle to be approximately $125 \mathrm{GeV}$ [3] with an uncertainty of $<0.2 \%$. Studies of its spin and parity have found it to be compatible with a $J^{P}=0^{+}$state in bosonic decays[4],[5]. Combined coupling fits of the measured production and decay rates have found no significant deviations from the Standard Model (SM) expectations [6]. These results strongly suggest that the newly discovered particle is indeed a Higgs boson and that a non-zero vacuum expectation value of a Higgs doublet is responsible for electroweak (EW) symmetry breaking[7],[8],[9]. In the Standard Model, all properties of the Higgs boson are defined once its mass is known. However, this model has many open questions such as the hierarchy problem regarding the naturalness of the Higgs boson mass and the nature of the dark matter. Many alternative theories addressing these issues make different predictions for the properties of one or more Higgs bosons. Indeed a crucial question is whether there is only one Higgs doublet, as postulated by the SM, or whether the Higgs sector is modified with respect to the SM, for example with a second doublet leading to more than one Higgs boson of which one has SM-like properties, as predicted in many beyondStandard-Model (BSM) theories. Precise measurements in the Higgs sector are therefore a high priority in the future program of particle physics.

Studying the new boson and probing for new physics beyond the SM, will require much more data to be acquired from collisions at a higher energy over a long period of time. In order to meet this challenge, innovations to the LHC complex will be undertaken in several phases, which will allow the reach of its physics program to be significantly extended. Following the first scheduled shutdown (LS1) during 2013-2014, the accelerator energy was increased to close to the design energy of $7 \mathrm{TeV}$ per colliding beam. Almost doubling the collision energy allows for a large jump in sensitivity to new particle states with higher mass including other, more massive, Higgs bosons. At the same time the instantaneous luminosity is approaching the design value of $1 \times$ $10^{34} \mathrm{~cm}^{-2} \mathrm{~s}^{-1}$. After the second scheduled long shutdown (LS2) in 2019-2020, the instantaneous luminosity will be increased by at least another factor of two allowing the LHC experiments to collect approximately ten times more data per year as compared to the rate achieved by the end of 2012. This will allow the collection of about $300 \mathrm{fb}^{-1}$ of integrated luminosity by about 2022 . This represents the target dataset size for the originally envisaged LHC experimental program. However, there is now a program underway to extend the LHC operations and further increase the luminosity to up to 7.5 times the design value, to allow the size of the dataset to be increased to $3000 \mathrm{fb}^{-1}$, over about a ten-year period starting in mid-2026. This phase of the experimental program is referred to as the High-Luminosity LHC, or HL-LHC and will commence after another scheduled long shutdown in 2024-2026 (LS3)[10].

The ATLAS detector[11] design for the high luminosity phase is well advanced and focuses on implementing pileup mitigation techniques. It will nevertheless require a significant amount of time to adapt and optimize the event reconstruction software to the high pileup conditions. The goal is that the performance of the new detector in the harsh conditions of the high luminosity phase will not be worse than the performance of the current detector with an average pileup of $\mu \approx 20$. For the studies presented in this note, the particle level quantities were modified by applying efficiency 
and resolution ("smearing") functions to physics objects[10],[12]. The pileup assumption varies from $\langle\mu>\approx 140$ to as high as $\langle\mu>\approx 200$.

\section{Higgs Couplings and Probing for Physics Beyond the Standard Model}

The luminosity of the HL-LHC will provide substantially improved statistical precision for already established channels and allow rare Higgs-boson production and decay modes to be studied. Assuming a Standard Model Higgs boson with a mass of $125 \mathrm{GeV}$, its coupling scale factors are determined from a combined fit to a series of Higgs decay channels, where the product $\sigma \cdot B(i \rightarrow$ $H \rightarrow f$ ) of cross-section and branching ratio for all contributing Higgs decay channels is expressed as a function of the coupling scale factors $\kappa_{i}[13]$. The contributing channels include:

\section{ATLAS Simulation Preliminary}

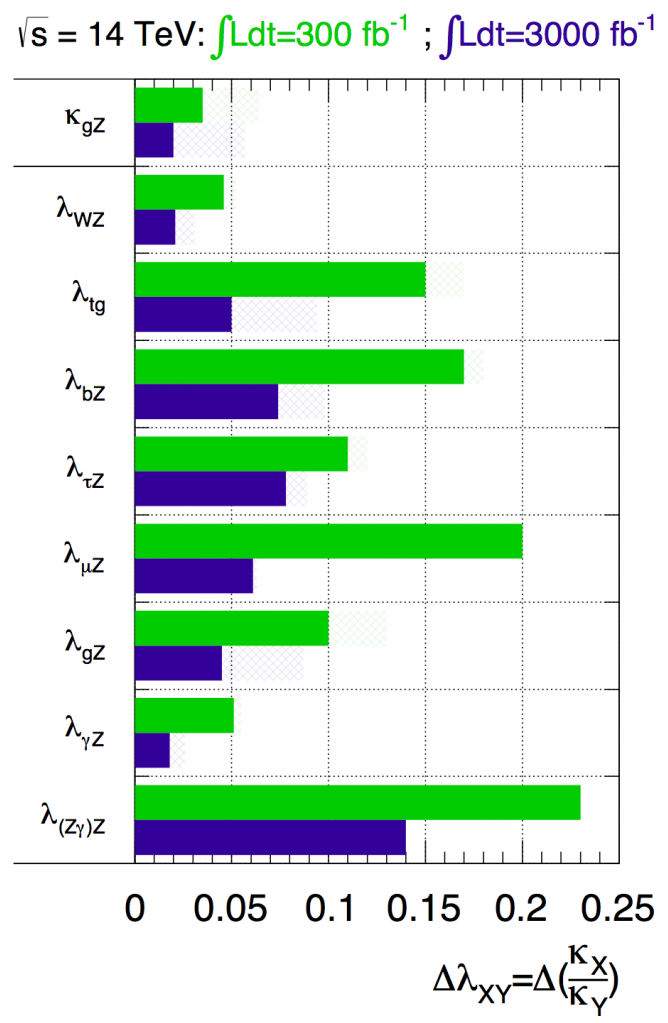

Figure 1: Relative uncertainty expected for the determination of coupling scale factor ratios $\lambda_{X Y}$ in a generic fit without assumptions, assuming a SM Higgs boson with a mass of $125 \mathrm{GeV}$ using $300 \mathrm{fb}^{-1}$ or $3000 \mathrm{fb}^{-1}$ of $14 \mathrm{TeV}$ LHC data. The hashed areas indicate the increase of the estimated error due to current theory systematic uncertainties[13].

1. $h \rightarrow \gamma \gamma$ in the inclusive, 0 -jet, 1-jet and 2-jet final states, the last one with a vector-boson fusion (VBF) selection as well as 1- and 2-lepton selections

2. $h \rightarrow Z Z^{(*)} \rightarrow 4 \ell$ separated into categories sensitive to gluon fusion (ggF), VBF, $Z h / W h$ and $t \bar{t} h$ production 
3. $h \rightarrow W W^{(*)} \rightarrow l v l v$ in the 0 -jet, 1 -jet and 2 -jet final states, the last with a VBF selection

4. $h \rightarrow Z \gamma, Z \rightarrow l l$ in inclusive and 2-jet (VBF) final states

5. $h \rightarrow \tau^{+} \tau^{-}$in the 2-jet final state with a VBF selection

6. $h \rightarrow \mu^{+} \mu^{-}$in inclusive and $t \bar{t} h$ final states

7. $h \rightarrow b \bar{b}$ with one-lepton and two-lepton final states to identify $Z h$ and $W h$ production respectively

When the assumption of the total width is removed and no other assumption is made, only ratios of coupling scale factors $\lambda_{X Y}=\kappa_{x} / \kappa_{Y}$ can be determined at the LHC. This representation has the additional advantage of the cancelation of many theoretical and experimental uncertainties. An example of such coupling ratio measurements under the assumption that no new particles are contributing though loops is shown in Fig. 1. For the majority of these coupling ratios, the precision without theory uncertainties is significantly improved by more than a factor of 2 with $3000 \mathrm{fb}^{-1}$ compared to $300 \mathrm{fb}^{-1}$.

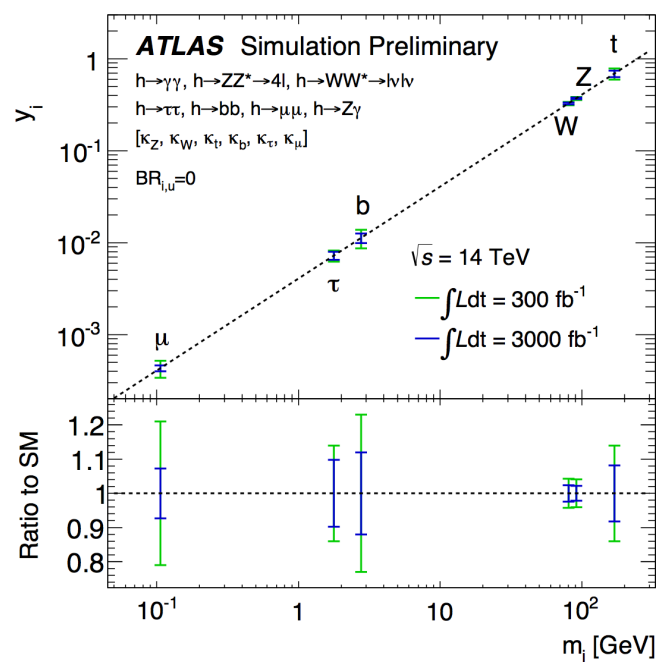

Figure 2: Fit results for the reduced coupling scale factors $y_{i}$ as a function of the particle mass, assuming $300 \mathrm{fb}^{-1}$ or $3000 \mathrm{fb}^{-1}$ of $14 \mathrm{TeV}$ data and a SM Higgs boson with a mass of $125 \mathrm{GeV}$. The diagonal, dashed line indicates the predicted mass dependence for the SM Higgs boson[13].

To test the predicted relationship between the Higgs boson couplings and the SM particle masses, reduced coupling scale factors $y_{i}$ are defined

$$
y_{V, i}=\sqrt{\kappa_{V, i} \frac{g_{V, i}}{2 v}}=\sqrt{\kappa_{V, i}} \frac{m_{V, i}}{v}
$$

for the weak bosons $V=W / Z$ (indexed by i) and

$$
y_{F, i}=\kappa_{F, i} \frac{g_{F, i}}{\sqrt{2}}=\kappa_{F, i} \frac{m_{F, i}}{v}
$$


for the fermions $F=\mu, \tau, b$ and $t$ (indexed by i) and where $g_{V, i}$ and $g_{F, i}$ denote the absolute gauge couplings to various bosons and Yukawa couplings to various fermions, respectively. Fig. 2 shows the reduced scale factors $y_{i}$ as a function of the mass of particle $\mathrm{i}$.

One can in addition probe for new physics beyond the Standard Model through the Higgs boson coupling studies[14]. Expected constraints on a non-standard mass scaling of the couplings, as well as on models with an additional electroweak singlet, an additional electroweak doublet (twoHiggs-doublet model), a composite Higgs boson, a simplified Supersymmetric Standard Model (MSSM) and a Higgs portal to dark matter, can also be derived by using the estimated precision on the coupling measurements with 300 and $3000 \mathrm{fb}^{-1}$ of collision data at $\sqrt{s}=14 \mathrm{TeV}$. The sensitivity to new phenomena is significantly enhanced at the HL-LHC compared to the corresponding sensitivities at lower integrated luminosities. Of interest are the two-Higgs-doublet models (2HDMs) which predict the existence of five Higgs bosons: two neutral CP-even bosons $h$ and $H$, one neutral CP-odd boson $A$ and two charged bosons $H^{ \pm}$. Both Higgs doublets acquire vacuum expectation values $v_{1}$ and $v_{2}$ respectively. Their ratio is denoted by $\tan \beta=v_{2} / v_{1}$ and they satisfy $v_{1}^{2}+v_{2}^{2}=v^{2} \approx(246 \mathrm{GeV})^{2}$. The Higgs sector of the $2 \mathrm{HDM}$ model can be described by six parameters: four Higgs boson masses $\left(m_{h}, m_{H}, m_{A}, m_{H^{ \pm}}\right), \tan \beta$ and the mixing angle $\alpha$ of the two neutral, CP-even Higgs states. Gauge invariance fixes the couplings of the two neutral, CP-even Higgs bosons to vector bosons relative to their SM values to be:

$$
\begin{aligned}
& g_{h V V}^{2 H D M} / g_{h V V}^{S M}=\sin (\beta-\alpha) \\
& g_{H V V}^{2 H D M} / g_{H V V}^{S M}=\cos (\beta-\alpha)
\end{aligned}
$$

Here $V=W, Z$ and $g_{h V V, H V V}^{S M}$ denote the SM Higgs boson couplings to vector bosons. The Higgsboson rate measurements in different production and decay modes are interpreted by assuming that the observed new particle with mass $m_{h} \sim 125 \mathrm{GeV}$ is the light CP-even neutral Higgs boson $h$. Shown in Fig. 3 are the regions of the $(\cos (\beta-\alpha), \tan \beta)$ plane that are expected to be excluded at $95 \% \mathrm{CL}$ or greater assuming the SM Higgs sector. The expected exclusion regions depend on the particular functional dependence of the couplings on $\beta$ and $\alpha$ which in Fig. 3 are for the lepton-specific Type-III 2HDM model. The SM-like alignment limit is at $\cos (\beta-\alpha)=0$.

Many Higgs portal models introduce an additional weakly-interacting massive particle (WIMP) as a dark matter candidate. It is assumed to interact very weakly with the SM particles, except for the Higgs boson. One can then measure the expected upper limit on the branching ratio of the Higgs boson to invisible final states, $B R_{i}$, derived using a combination of rate measurements. The Higgs-boson production modes are assumed to be those prescribed by the SM and couplings of the Higgs to massive particles other than the WIMP are assumed to be equal to the SM predictions. To compare with direct searches for dark matter, the expected upper limit on $B R_{i}$ is translated into constraints on the coupling of the WIMP to the Higgs boson as a function of its mass. It is assumed that the WIMP mass is less than half the Higgs-boson mass and that the resulting Higgs-boson decays to WIMP pairs account entirely for $B R_{i}$. These assumptions produce conservative limits as any additional contributions to $B R_{i}$ from other new phenomena would produce more stringent results. WIMP candidates can be scalar, Majorana fermion or vector dark matter particles. The coupling is then re-parameterized in terms of cross-section for scattering between the WIMP and nucleons via Higgs boson exchange $\sigma_{\chi-N}[15]$. Expected upper limits at $95 \% \mathrm{CL}$ on $\sigma_{\chi-N}$ are 


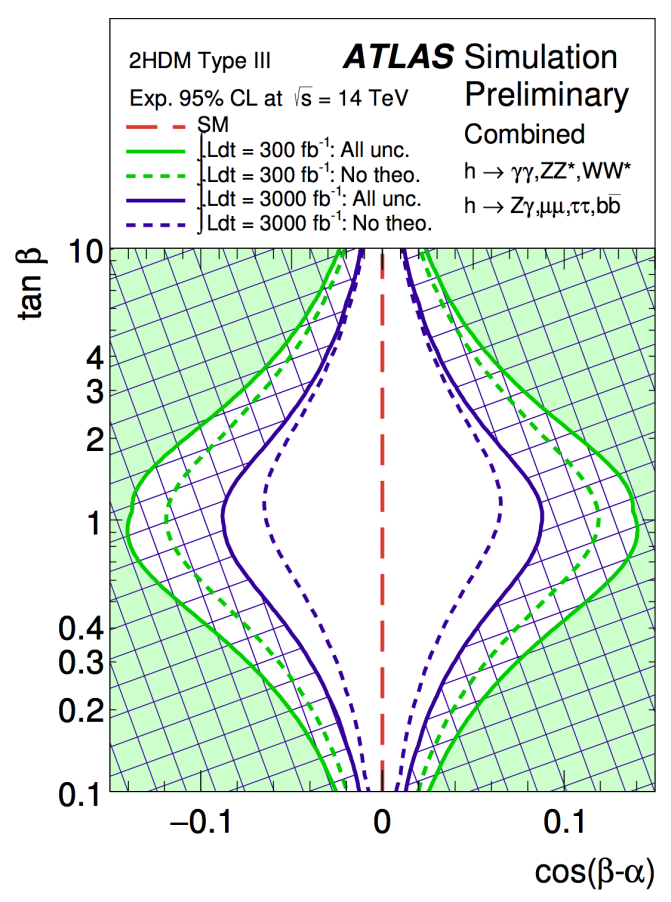

Figure 3: Regions of the $(\cos (\beta-\alpha), \tan \beta)$ plane expected to be excluded by fits to the measured rates of Higgs boson production and decays. The confidence intervals account for a possible relative sign between different couplings. The expected likelihood contours correspond approximately to 95\% CL ( $2 \sigma)$ and are indicated assuming the SM Higgs sector. The light shaded and hashed regions indicate the expected exclusions[14].

derived as a function of the WIMP mass $m_{\chi}(\chi=S, V, f)$. The results expected for $3000 \mathrm{fb}^{-1}$ of data at $\sqrt{s}=14 \mathrm{TeV}$ including all systematic uncertainties are shown in Fig. 4.

\section{Higgs Production via Vector Boson Fusion}

In the SM, vector-boson fusion (VBF) is a production mechanism wherein two incoming quarks emit virtual $\mathrm{W}$ or $\mathrm{Z}$ bosons which undergo inverse pair decay to form a Higgs boson. As shown in Fig. 5, contributions to leading order VBF production are made in the $s, t$ and $u$ channels. However, at circular hadron colliders such as the HL-LHC, the $t$ and $u$ fusion channels are heavily favored[16]. It is worth noting as well, that the $W W$ fusion contribution is the dominant term in the VBF cross-section, a consequence of the larger coupling of the $W$ boson to fermions[17]. VBF Higgs-boson production is predicted to be the second largest contribution to the total Higgs-boson production cross-section for a mass $m_{H} \approx 125 \mathrm{GeV}$, though it is still an order of magnitude smaller than the production cross-section for gluon fusion. Unlike the gluon fusion process though, the VBF production signature is highly distinctive, marked by the presence of two highly energetic final state quark jets at leading order, which tend to be found in opposite forward regions of cylindrical detectors such as ATLAS. Furthermore, the resultant Higgs boson tends to be produced in the central region, with low momentum, such that $E_{H} \approx m_{H}$. As such, the distinct kinematic properties of the dijet and Higgs systems can be used to simultaneously reduce backgrounds originating 


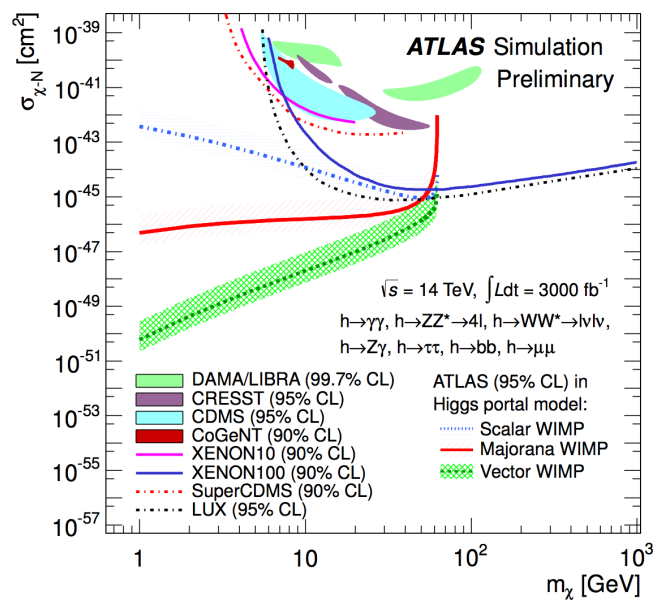

Figure 4: ATLAS expected upper limit at 95\% CL on the WIMP-nucleon scattering cross section in a Higgs portal model as a function of the mass of the dark matter particle, shown separately for a scalar, Majorana fermion, or vector boson WIMP, with $3000 \mathrm{fb}^{-1}$ of data at $\sqrt{s}=14 \mathrm{TeV}$ and including all systematic uncertainties. The hashed bands indicate the uncertainty resulting from the form factor $f_{N}$. Excluded and allowed regions from direct detection experiments at the confidence levels indicated are also shown. These are spin-independent results obtained directly from searches for nuclei recoils from elastic scattering of WIMPs, rather than being inferred indirectly through Higgs boson exchange in the Higgs portal model[14].

in QCD interactions and identify VBF-like events. Pileup mitigation both at the detector and data analysis levels are extremely crucial. The VBF Higgs-boson production hence can be used to investigate the impact of increased tracking coverage in the forward direction in terms of the separation of VBF and gluon fusion-mediated Higgs-boson production in association with two jets and in the consequent pileup suppression. Three forward ATLAS tracker scenarios have been investigated: the reference scenario $(|\eta| \leq 4.0)$; the middle scenario $(|\eta| \leq 3.2)$; and the low scenario $(|\eta| \leq 2.5)[10]$. In this section results from two benchmark processes are presented.
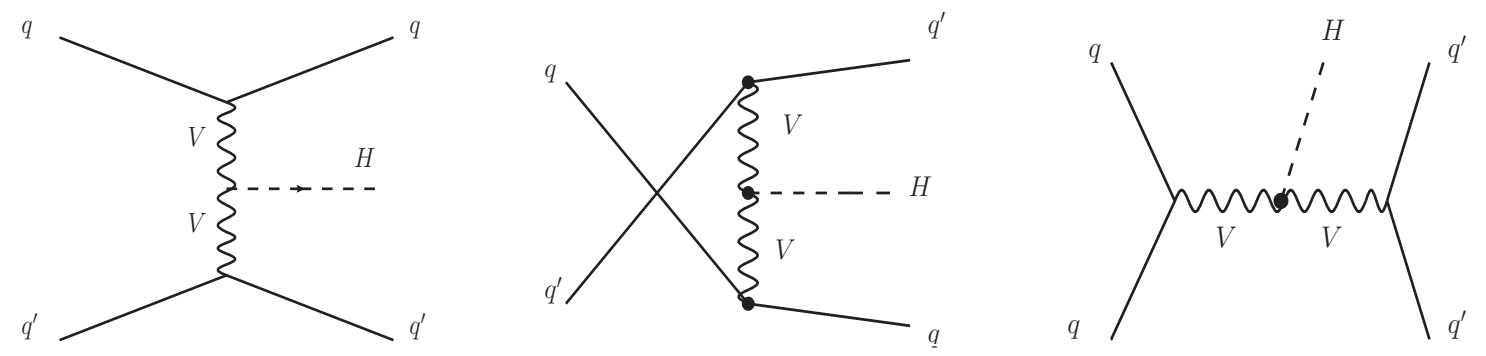

Figure 5: Leading order diagrams for the Higgs boson production through vector-boson fusion. Here, $V=W^{ \pm}$or $Z$.

The $H \rightarrow Z Z^{(*)} \rightarrow 4 \ell$ decay channel and in particular in a final state involving two energetic jets, is relatively clean and any non-Higgs related background sources other than from pileup contributions is negligible and can be discarded[18]. The main objectives are to efficiently separate VBF from gluon fusion events with two jets in the final state and to provide a VBF-like signal with 
as little contamination from pileup as possible. To achieve this, a Boosted Decision Tree (BDT) approach is used based on the following kinematic variables: invariant mass of the selected forward jets, $m_{j j}$; separation of these jets in $\eta, \eta_{j j}$; transverse momentum of the Higgs boson plus jets, $p_{T, H j j}$; transverse momentum of each of the jets, $p_{T, j e t 1}$ and $p_{T, j e t 2}$; and the Zeppenfeld variable $\eta_{H}^{Z e p p}=\eta_{Z Z}-<\eta_{j e t 1}, \eta_{\text {jet } 2}>$. In Fig. 6 the resulting BDT output for the reference, middle, and low scenarios is shown. Events are then divided into three categories defined by a selection on the BDT output with bin edges $[1,0.8,0.6,0]$ and the negative log likelihood (NLL) minimization technique is used to fit in all three bins simultaneously for the signal significance $Z_{0}$ and $\Delta \mu$. It
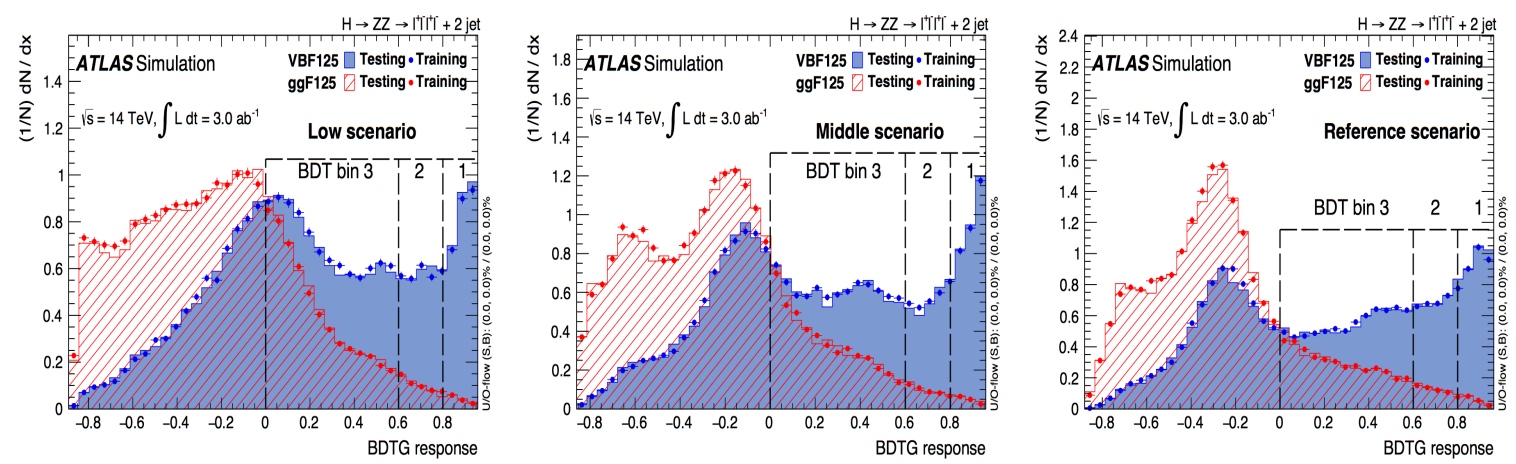

Figure 6: BDT classifier distributions for Low, Middle, and Reference detector layouts at $\langle\mu\rangle=200$ [18].

is observed that $Z_{0}$ decreases by $2 \%$ and $12 \%$ in the middle and low scenarios, respectively, and the signal strength uncertainty $\Delta \mu / \mu$ increases by $4 \%$ and $11 \%$ when theoretical uncertainties are considered, all with respect to the reference scenario. Although the VBF production mechanism will very likely have been discovered in the $H \rightarrow 4 \ell$ channel by the timescale of the HL-LHC, significantly high signal event counts will still be required to perform precision measurements of Higgs-boson properties, such as couplings and the potential probing of BSM physics in the Higgs sector, which justify the emphasis given in the analysis presented here on obtaining a VBF signal region with the smallest possible contamination.

An important benchmark for the VBF Higgs-boson production mechanism is provided by the $H \rightarrow \tau^{+} \tau^{-}$decay channel[19]. In addition to the effective suppression of pileup in the forward region, identifying the VBF-like signal requires excellent $E_{T}^{m i s s}$ resolution. Both provide strong motivations for an extended pseudorapidity coverage of the ATLAS inner tracker. For the results presented in this note, one tau decays leptonically and the other hadronically. Dominant background sources include $Z \rightarrow \tau^{+} \tau^{-}$, fake taus and contributions from the $t \bar{t}$ and single-top production. The contamination of pileup jets in the VBF channel for the total background is significant. Most events (72\%) have a sub-leading pileup jet and nearly half (42\%) have a leading pileup jet. These pileup jets are especially problematic because they are typically forward. To discriminate VBF-like signal events from all background sources a BDT approach is used, similar to the one used in the $H \rightarrow 4 \ell$ case. Shown in Fig. 7 is the efficiency for rejecting background versus the efficiency for selecting signal at $90 \%$ forward pileup rejection for the three inner tracker extension scenarios (reference, medium, low). For the reference scenario it is expected that the signal strength uncertainty $\Delta \mu / \mu$ will be $\sim 8 \%$ for a $10 \%$ systematic uncertainty on the backgrounds and 
a 5\% experimental uncertainty on the observed signal. The theoretical uncertainties on the signal have been ignored.

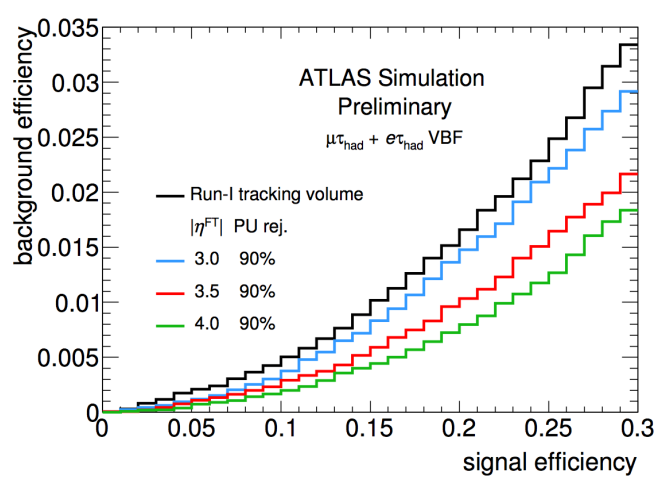

Figure 7: Signal efficiency versus background efficiency for scenarios of generic forward tracker coverage and rejection power. A BDT is trained in the VBF category for each scenario[19].

\section{Rare Higgs-boson decays: $H \rightarrow \mu^{+} \mu^{-}$}

The $H \rightarrow \mu^{+} \mu^{-}$is a most sensitive channel where couplings to second generation fermions can be measured at the HL-LHC with a clean final-state signature. Due to the high resolution of the reconstructed $\mu^{+} \mu^{-}$invariant mass, it can also contribute to the Higgs mass measurements. The branching ratio for the $H \rightarrow \mu^{+} \mu^{-}$decays is of the order $27.6-6.2 \times 10^{-5}$ in the Higgs boson mass range of 110-150 GeV[20]. A dominant irreducible SM background is the $Z / \gamma^{*} \rightarrow \mu^{+} \mu^{-}$ process with a very high production rate making this a challenging measurement with the currently recorded data. Possible extensions of SM, such as the Minimal Supersymmetric Extension (MSSM), postulate the existence of additional Higgs boson-like particles. For large parts of the allowed parameter spaces in the MSSM couplings between Higgs bosons and down-type fermions are enhanced leading to an increased branching fraction of the $H \rightarrow \mu^{+} \mu^{-}$decays. In what follows the sensitivity of the ATLAS detector to this decay for a SM Higgs-boson and for an integrated luminosity of $3000 \mathrm{fb}^{-1}$, is presented[21]. The pileup is assumed to be $\langle\mu\rangle \approx 140$.

A $\mu^{+} \mu^{-}$pair is chosen from the two highest $p_{T}$ muons having opposite charge. Pairs with an invariant mass $m_{\mu^{+} \mu^{-}}>70 \mathrm{GeV}$ are selected. The resulting $\mu^{+} \mu^{-}$invariant mass distribution of the signal and background processes are shown in Fig. 8. Even for invariant masses well beyond the $Z$ resonance, the $Z / \gamma^{*}$ production dominates the background. In the mass range $m_{\mu^{+}} \mu^{-}>110 \mathrm{GeV}$ its relative fraction amounts to $80.6 \%$, with $t \bar{t}$ and $W W$ accounting for almost all of the remaining contribution. The expected signal significance and the precision on the signal strength $\Delta \mu / \mu$ are obtained from the signal and background distributions in the full fit range of $100 \mathrm{GeV}$ to $160 \mathrm{GeV}$ taking into account the signal and background shapes. With an integrated luminosity of $3000 \mathrm{fb}^{-1}$, the $H \rightarrow \mu^{+} \mu^{-}$decay can be observed with an expected significance of $7.0 \sigma$. The corresponding signal strength uncertainty is $\Delta \mu / \mu=21 \%$.

Of particular interest is the associated Higgs-boson production with a pair of top quarks and its subsequent decay into a pair of muons. A study of this rare channel has two motivations. First, 

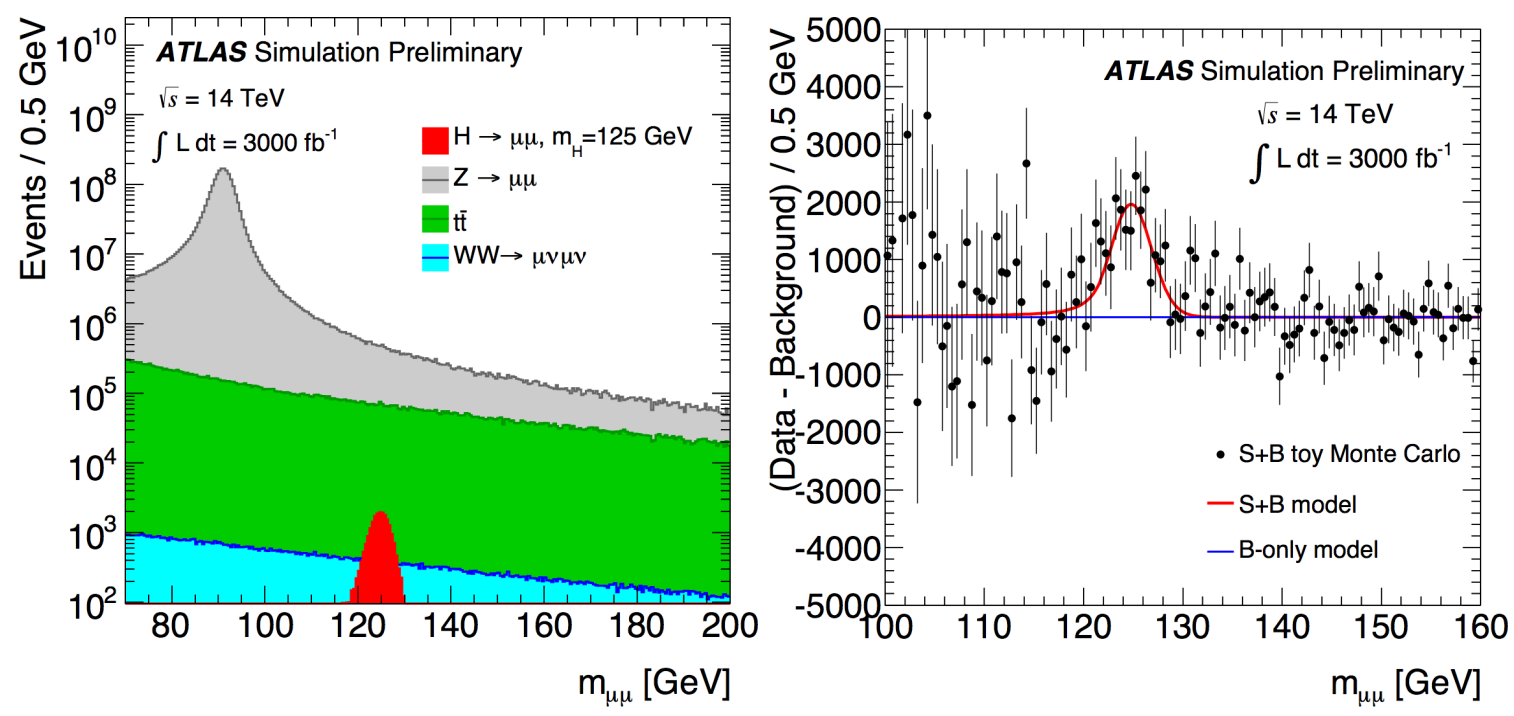

Figure 8: Left: Distribution of the $\mu^{+} \mu^{-}$invariant mass of the signal and background processes generated for $\sqrt{s}=14 \mathrm{TeV}$ and $3000 \mathrm{fb}^{-1}$ integrated luminosity. Right: Background subtracted invariant mass distribution of a toy MC sample generated under the signal-plus-background hypothesis for $3000 \mathrm{fb}^{-1}$ [21].

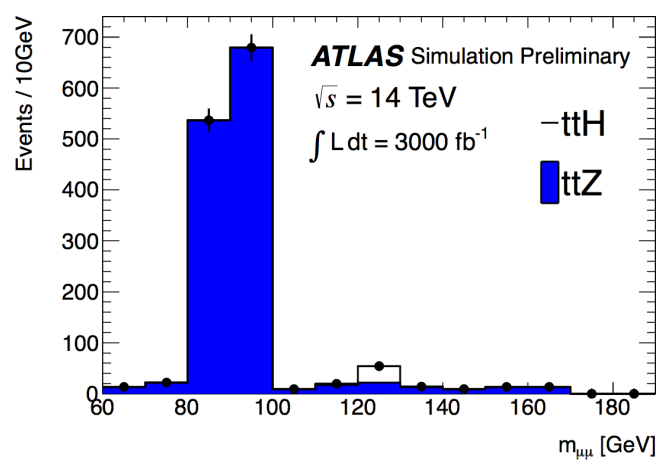

Figure 9: The invariant mass of the di-muon system in the $t \bar{t} H, H \rightarrow \mu^{+} \mu^{-}$channel[21].

it allows a direct measurement of the product of the top- and the $\mu$-Yukawa couplings, neither of which are accessible through the standard Higgs channels. Second, this channel could be valuable for the determination of the $\mathrm{CP}$ nature of the resonance at $125 \mathrm{GeV}$. The $\mathrm{CP}$-odd component could be suppressed with a vector-boson coupling in the initial or final state, but there are only fermion Yukawa couplings in this channel. Candidate signal events must have at least two muons with opposite charge and $p_{T}>35 \mathrm{GeV}$, no more than four leptons, at least four jets and a Higgs candidate mass formed from the two muons with $m_{H}$ between $120 \mathrm{GeV}$ and $130 \mathrm{GeV}$. The distribution of the di-muon mass is shown in Fig. 9. The expected number of events after all the selections is 33 for signal and 22 for background, allowing this channel to be observed with the HL-LHC. 


\section{Higgs boson pair production}

The Higgs boson pair production is an important part of the HL-LHC experimental program. It allows for the measurement of the Higgs trilinear self-coupling $\lambda_{H H H}$ and the subsequent reconstruction of the Higgs potential which will in its turn provide a strong confirmation of whether the Higgs mechanism is responsible for the electroweak symmetry breaking. For a center-of-mass energy of $14 \mathrm{TeV}$, the production cross-section of pairs of $125 \mathrm{GeV}$ Higgs bosons is estimated to be $40.8 \mathrm{fb}$ (with an error of $\pm 8.5 \%$ from QCD scale uncertainties and $\pm 7 \%$ from $\mathrm{PDF}+\alpha_{s}$ )[22],[23].
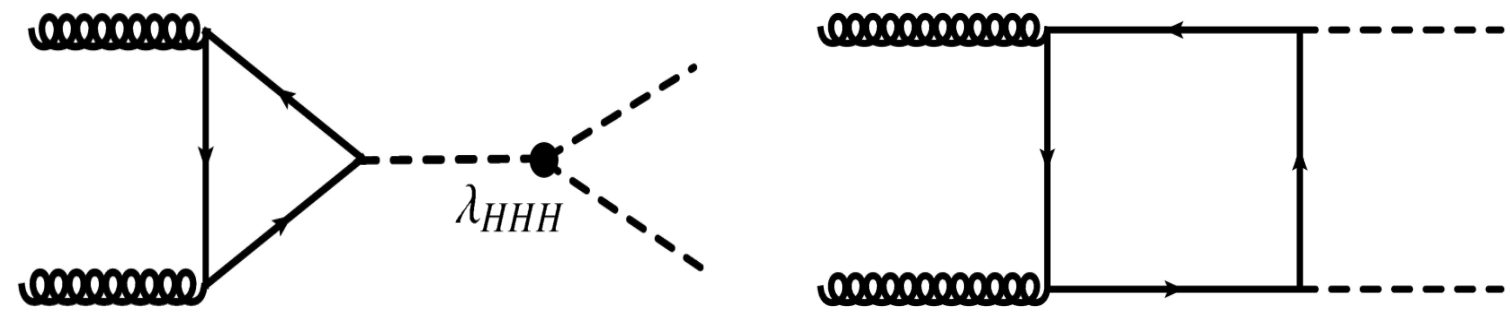

Figure 10: Feynman diagrams describing Higgs pair production from gluon fusion at leading order.

Higgs boson pair production is dominated by gluon fusion and can be described at leading order by the Feynman diagrams shown in Fig. 10. Only the diagram on the left side includes a contribution from the triple Higgs coupling, whereas in the case of the diagram on the right hand side the self-coupling constant does not play a role. There is a relative minus sign between the two contributions, resulting in destructive interference that effectively reduces the total Higgs pair production cross-section in the SM. A value of $\lambda_{H H H}=0$ corresponds to the case where there is no self-coupling of the Higgs boson and thus the amplitude of the left diagram in Fig. 10 vanishes. For this case the cross-section is enhanced by approximately a factor of two compared to the SM[24]. The cross-section decreases with increasing values of the self-coupling up to a value of 2.44 times the SM value where the cross-section reaches its minimum. For larger values of $\lambda_{H H H}$ the crosssection increases again. For a data sample of $3000 \mathrm{fb}^{-1}$, the expected yields in the available final states range from 1 in the case of $\gamma \gamma \gamma \gamma$ to about 41,000 for $b \bar{b} b \bar{b}$. Experimentally, the two most promising modes are $b \bar{b} \gamma \gamma$ and $b \bar{b} \tau \tau$ with expected yields of 320 and 8900 events, respectively. These are discussed further in this note.

Despite the low branching ratio, the $H(\rightarrow b \bar{b}) H(\rightarrow \gamma \gamma)$ final state[25] offers the potential for a clean $H H$ signal extraction thanks to the narrow mass peak of the $H \rightarrow \gamma \gamma$ decay. There are several processes which contribute irreducible backgrounds in this channel, since they include two real photons and two real jets in the final state, such as $b \bar{b} \gamma \gamma, t \bar{t} H(\gamma \gamma), Z(b \bar{b}) H(\gamma \gamma)$ and $b \bar{b} H(\gamma \gamma)$. Significant reducible background contributions are also possible from final states in which one or more objects are misidentified, such as a light flavor jet or $c$-jet being mis-identified as a $b$-jet or an electron or light flavor jet being misidentified as a photon. To optimize the signal selection, the reconstructed invariant mass of the two-jet and the two-photon systems are constrained within $100-150 \mathrm{GeV}$ and $123-128 \mathrm{GeV}$, respectively. Additional cuts on the angular separation between the two photons, the two $b$-jets as well as the minimum separation between a photon and a $b$-jet are applied. Following these selections, an expected signal yield of 8.4 events in $3000 \mathrm{fb}^{-1}$ is obtained. The expected total background yield is 47 events with the largest contributions coming from $b \bar{b} \gamma \gamma$ 


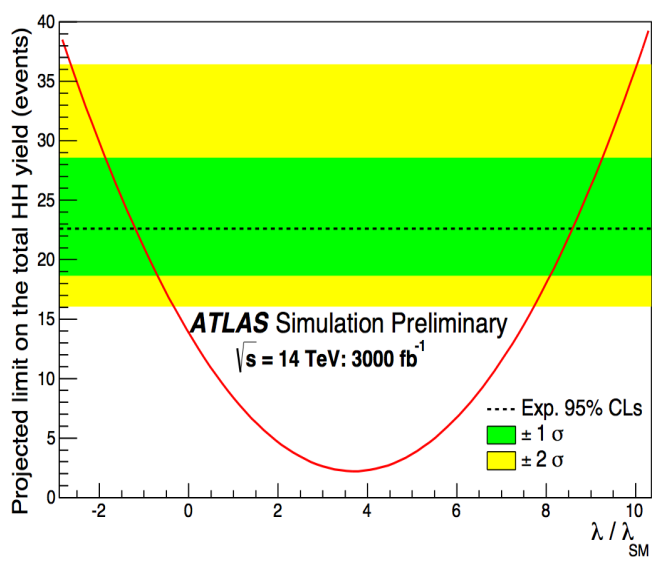

Figure 11: $95 \% \mathrm{CL}_{s}$ limit (dashed line) on the size of an additional $H H$ contribution summed with the expected Standard Model $H H$ yield, as well as the $\pm 1 / 2 \sigma$ uncertainty bands, overlaid on the number of predicted total (box + self-coupling) $H H$ events as a function of $\lambda / \lambda_{S M}$ after application of the analysis cuts (solid line)[25].

and $b \bar{b} \gamma j$ events. This is in the presence of $\left\langle\mu>\approx 140\right.$ pileup. Using the $\mathrm{CL}_{s}$ technique one can then set an upper limit on the total (box + self-coupling) number of $H H$ signal events corresponding to $3000 \mathrm{fb}^{-1}$ and as a function of $\lambda / \lambda_{S M}$. An exclusion at $95 \% \mathrm{CL}$ of BSM models with $\lambda / \lambda_{S M} \leq-$ 1.3 and $\lambda / \lambda_{S M} \geq 8.7$ is derived. This is shown on Fig. 11 overlaid on the number of predicted $H H$ events as a function of $\lambda / \lambda_{S M}$.

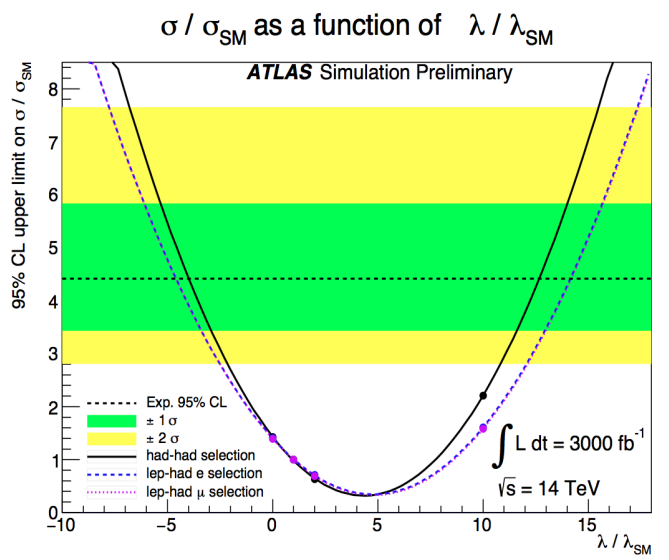

Figure 12: The 95\% CL upper limit on the cross section of the $H H \rightarrow b \bar{b} \tau^{+} \tau^{-}$assuming Standard Model couplings is shown in the dashed line with the $68 \%$ and $95 \%$ error bands. The solid black, dashed blue and dotted violet lines show a fit of the expected number of events normalized by the SM number of events for different $\lambda_{H H H}$ after the selection for the $\tau_{\text {had }} \tau_{\text {had }}, \tau_{\text {lep }} \tau_{\text {had }}$ electron and muon channels[26].

The process $H(\rightarrow b \bar{b}) H(\rightarrow \tau \tau)$ results in a significantly larger yield due to the larger branching ratio of the $H \rightarrow \tau^{+} \tau^{-}$decay when compared to the $H \rightarrow \gamma \gamma$ one[26]. The downturn is the more complex final state and the larger backgrounds which make the determination of the signal yield (and strength) more challenging. Nevertheless this process can still provide a confirmation of the di- 
Higgs production and perhaps shed some light on the size of the Higgs trilinear self-coupling $\lambda_{H H H}$. All the decay modes of the $\tau$ leptons can be included: $\tau_{\text {lep }} \tau_{\text {lep }}, \tau_{\text {lep }} \tau_{\text {had }}$ and $\tau_{\text {had }} \tau_{\text {had }}$, where $\tau_{\text {lep }}$ means a $\tau$ which decays into a $\mu$ or $e$ lepton plus a pair of neutrinos and $\tau_{\text {had }}$ represents a $\tau$ decaying into a $v_{\tau}$ plus hadrons. As it turns out the fully leptonic final state does not really contribute to the final result. There are several common irreducible background sources for all these sub-channels, the most dominant ones being the $t \bar{t}$ and the $Z(\tau \tau)+$ jets ones. The cut-based selection procedure depends on the $\tau$-lepton decay mode and is optimized for maximum signal to background ratio. In all cases the selected events are required to have two reconstructed leptons within a $m_{\tau \tau}$ window whose reconstruction efficiency significantly depends on the amount of pileup (here $\langle\mu>\approx 140$ ) present. The combined signal strength for all $\tau$ decay modes when $\lambda_{H H H} / \lambda_{S M}=1$, is $\sim 0.6$ and it rises to 1.14 for $\lambda_{H H H} / \lambda_{S M}=10$. Using these results one can set a 95\% CL upper limit on the cross-section $H H \rightarrow b \bar{b} \tau^{+} \tau^{-}$as a function of $\lambda_{H H H} / \lambda_{S M}$. This is shown in Fig. 12. An exclusion at $95 \% \mathrm{CL}$ of BSM models with $\lambda / \lambda_{S M} \leq-4$ and $\lambda / \lambda_{S M} \geq 12$ can then be derived.

\section{Summary}

The HL-LHC provides an unprecedented opportunity to study the Higgs boson in great detail and offers a unique ability to probe new physics beyond the Standard Model. The expected physics reach of the ATLAS detector at the HL-LHC era and its discovery potential in the Higgs sector, has been the subject of a series of studies that commenced in earnest as part of the European Strategy discussions in 2012. These studies have continued, and been refined, through the 2013 Snowmass process, the EFCA HL-LHC workshops in 2013 and 2014, and then the detector scoping exercise undertaken in 2015. Initial studies were mainly done for an instantaneous luminosity of $5 \times 10^{34} \mathrm{~cm}^{2} \mathrm{~s}^{-1}$ corresponding to $\langle\mu\rangle=140$. For the Phase-II detector scoping exercises the experiments assumed a maximum instantaneous luminosity of $7.5 \times 10^{34} \mathrm{~cm}^{2} \mathrm{~s}^{-1}$ corresponding to $<\mu>=200$. Some of the Higgs-related analyses have been presented in this note. Further studies will be undertaken once ATLAS has fully defined its Phase-II detector configuration.

\section{References}

[1] ATLAS Collaboration, Observation of a new particle in the search for the Standard Model Higgs boson with the ATLAS detector at the LHC, Phys. Lett. B 716 (2012) 1-29.

[2] CMS Collaboration, Observation of a new boson at a mass of $125 \mathrm{GeV}$ with the CMS experiment at the LHC, Phys. Lett. B 716 (2012) 30-61.

[3] ATLAS Collaboration, CMS Collaboration, Combined Measurement of the Higgs Boson Mass in $p p$ Collisions at $\sqrt{s}=7$ and $8 \mathrm{TeV}$ with the ATLAS and CMS Experiments, Phys. Rev. Lett. 114 (2015) 191803.

[4] CMS Collaboration, Constraints on the spin-parity and anomalous HVV couplings of the Higgs boson in proton collisions at 7 and 8 TeV, Phys. Rev. D 92 (2015) 012004.

[5] ATLAS Collaboration, Study of the spin and parity of the Higgs boson in diboson decays with the ATLAS detector, Eur. Phys. J. C 75 (2015) 476.

[6] ATLAS Collaboration, CMS Collaboration, Measurements of the Higgs boson production and decay rates and constraints on its couplings from a combined ATLAS and CMS analysis of the LHC $p p$ collision data at $\sqrt{s}=7$ and $8 \mathrm{TeV}$, JHEP 1608 (2016) 045 . 
[7] F. Englert and R. Brout, Broken Symmetry and the mass of gauge vector mesons, Phys. Rev. Lett. 13 (1964) 321-323.

[8] P. W. Higgs, Broken Symmetries and the masses of gauge bosons, Phys. Rev. Lett. 13 (1964) 508-509.

[9] G. S. Guralnik, C. R. Hagen, and T. W. B. Kibble, Global conservation laws and massless particles, Phys. Rev. Lett. 13 (1964) 585-587.

[10] ATLAS Collaboration, ATLAS Phase-II Scoping Document, CERN-LHCC-2015-020

[11] ATLAS Collaboration, JINST 3 (2008) S08004.

[12] ATLAS Collaboration, Performance assumptions for an upgraded ATLAS detector at a High-Luminosity LHC, ATL-PHYS-PUB-2013-009 (2013), http://cds.cern.ch/record/1604420.

[13] ATLAS Collaboration, Projections for measurements of the Higgs boson signal strengths and coupling parameters with the ATLAS detector at the HL-LHC, ATL-PHYS-PUB-2014-016 (2014), http://cds.cern.ch/record/1956710.

[14] ATLAS Collaboration, Prospects for New Physics in Higgs Couplings Studies with the ATLAS Detector at the HL-LHC, ATL-PHYS-PUB-2014-017 (2014), http://cds.cern.ch/record/1956711.

[15] A. Djouadi, O. Lebedev, Y. Mambrini and J. Quevillon, Implications of LHC searches for Higgs-portal dark matter, Phys. Lett. B 709 (2012) 65-69.

[16] LHC Higgs Cross Section Working Group, Handbook of LHC Higgs Cross Sections: 1. Inclusive Observables, (2011), arXiv: 1101.0593 [hep-ph].

[17] A. Djouadi, The anatomy of electroweak symmetry breaking: Tome I: The Higgs boson in the Standard Model, Physics Reports 457 (2008) 1.

[18] ATLAS Collaboration, Prospective results for vector-boson fusion-mediated Higgs-boson searches in the four-lepton final state at the High Luminosity Large Hadron Collider, ATL-PHYS-PUB-2016-008 (2016), http://cds.cern.ch/record/2145377.

[19] ATLAS Collaboration, Studies of the VBF $H \rightarrow \tau_{l} \tau_{\text {had }}$ analysis at High Luminosity LHC conditions, ATL-PHYS-PUB-2014-018 (2014), http://cds.cern.ch/record/1956732.

[20] LHC Higgs Cross Section Working Group, S. Dittmaier, C. Mariotti, G. Passarino, and R. Tanaka (Eds.), Handbook of LHC Higgs cross sections: 2. Differential distributions, (2012), arXiv: 1201.3084 [hep-ph].

[21] ATLAS Collaboration, Projections for measurements of Higgs boson cross sections, branching ratios and coupling parameters with the ATLAS detector at a HL-LHC, ATL-PHYS-PUB-2013-014 (2013), http://cds.cern.ch/record/1611186.

[22] D. de Florian and J. Mazzitelli, Higgs Boson Pair Production at Next-to-Next-to-Leading Order in QCD, Phys. Rev. Lett. 111 (2013) 201801.

[23] J. Grigo, K. Melnikov and M. Steinhauser, Virtual corrections to Higgs boson pair production in the large top quark mass limit, Nucl. Phys. B888 (2014) 17-29.

[24] M. J. Dolan, C. Englert and M. Spannowsky, Higgs self-coupling measurements at the LHC, JHEP 1210 (2012) 112.

[25] ATLAS Collaboration, Prospects for measuring Higgs pair production in the channel $H(\rightarrow b \bar{b}) H(\rightarrow \gamma \gamma)$ using the ATLAS detector at the HL-LHC, ATL-PHYS-PUB-2014-019 (2014), http://cds.cern.ch/record/1956733.

[26] ATLAS Collaboration, Higgs Pair Production in the $H(\rightarrow b \bar{b}) H\left(\rightarrow \tau^{+} \tau^{-}\right)$channel at the High-Luminosity LHC, ATL-PHYS-PUB-2015-046 (2015), http://cds.cern.ch/record/2065974. 